\title{
Progressive polarity loss and luminal collapse disrupt tissue organization in carcinoma
}

\author{
Ruba Halaoui, ${ }^{1,2}$ Carlis Rejon, ${ }^{1}$ Sudipa June Chatterjee, ${ }^{1,2}$ Joseph Szymborski, ${ }^{1,2}$ Sarkis Meterissian, ${ }^{3,4,5}$ \\ William J. Muller, ${ }^{1,6}$ Atilla Omeroglu, ${ }^{5,7}$ and Luke McCaffrey ${ }^{1,2,3}$ \\ ${ }^{1}$ Rosalind and Morris Goodman Cancer Research Centre, McGill University, Montreal, Quebec H3A 1A3, Canada; ${ }^{2}$ Division of \\ Experimental Medicine, McGill University, Montreal, Quebec H4A 3J1, Canada; ${ }^{3}$ Department of Oncology, McGill University, \\ Montreal, Quebec H3A 0G4, Canada; ${ }^{4}$ Department of Surgery, McGill University, Montreal, Quebec H3G 1A4, Canada; ${ }^{5}$ McGill \\ University Health Centre, Montreal, Quebec H4A 3J1, Canada; ${ }^{6}$ Department of Biochemistry, McGill University, Montreal, \\ Quebec H3G 1Y6, Canada; ${ }^{7}$ Department of Pathology, McGill University, Montreal, Quebec H3A 2B4, Canada
}

Epithelial cancers (carcinoma) account for $\mathbf{8 0 \% - 9 0 \% ~ o f ~ a l l ~ c a n c e r s . ~ T h e ~ d e v e l o p m e n t ~ o f ~ c a r c i n o m a ~ i s ~ a s s o c i a t e d ~}$ with disrupted epithelial organization and solid ductal structures. The mechanisms underlying the morphological development of carcinoma are poorly understood, but it is thought that loss of cell polarity is an early event. Here we report the characterization of the development of human breast lesions leading to carcinoma. We identified a unique mechanism that generates solid ducts in carcinoma through progressive loss of polarity and collapse of the luminal architecture. This program initiates with asymmetric divisions of polarized cells that generate a stratified epithelium containing both polarized and depolarized cells. Stratified regions form cords that penetrate into the lumen, subdividing it into polarized secondary lumina. The secondary lumina then collapse with a concomitant decrease in RhoA and myosin II activity at the apical membrane and ultimately lose apical-basal polarity. By restoring RhoA activity in mice, ducts maintained lumen and cell polarity. Notably, disrupted tissue architecture through luminal collapse was reversible, and ducts with a lumen were re-established after oncogene suppression in vivo. This reveals a novel and common mechanism that contributes to carcinoma development by progressively disrupting cell and tissue organization.

[Keywords: epithelial; cancer progression; morphogenesis; breast cancer; duct]

Supplemental material is available for this article.

Received April 16, 2017; revised version accepted August 11, 2017.

Epithelial cells line many organ and tissue surfaces and are the origin of $80 \%-90 \%$ of all human cancers, called carcinoma. Carcinogenesis is the process in which normal epithelial cells progressively evolve to become cancer cells, which is associated with disrupted cell and tissue organization. However, the mechanisms that underlie the early development and progression of carcinoma remain poorly characterized.

Epithelial ducts are highly organized structures at both cellular and tissue levels, which are disrupted in carcinoma. Apical-basal membrane polarity demarcates different cellular membrane compartments, with an apical membrane that faces the lumen and a basolateral membrane that contacts adjacent cells and the extracellular matrix (Rodriguez-Boulan and Macara 2014). Multiple protein complexes cooperate to establish distinct membrane domains, which are essential to organize intracellular sig-

Corresponding author: luke.mccaffrey@mcgill.ca

Article published online ahead of print. Article and publication date are online at http://www.genesdev.org/cgi/doi/10.1101/gad.300566.117. naling pathways for proper growth control, tissue organization, and suppression of invasion and metastasis (Rodriguez-Boulan and Macara 2014; Halaoui and McCaffrey 2015). The Par complex consists of a core scaffold Par3 that recruits the adaptor protein Par6 and its effector kinase, aPKC. After being recruited by Par3, Par6 and aPKC dissociate from Par3 and associate with the Crumbs complex (Crb3/Pals1/Patj) at the apical membrane in mature epithelial cells (Rodriguez-Boulan and Macara 2014). The Scrib complex consists of Scribble, discs large 1 (Dlg1), and lethal giant larvae (Lg12) and acts to mutually restrict the spatial organization of the apical complexes (Rodriguez-Boulan and Macara 2014). Accumulating evidence indicates that apical-basal polarity is a barrier to carcinogenesis (Halaoui and McCaffrey 2015). For

(C) 2017 Halaoui et al. This article is distributed exclusively by Cold Spring Harbor Laboratory Press for the first six months after the full-issue publication date (see http://genesdev.cshlp.org/site/misc/terms.xhtml). After six months, it is available under a Creative Commons License (Attribution-NonCommercial 4.0 International), as described at http://creativecommons.org/licenses/by-nc/4.0/. 
example, depletion or mislocalization of individual polarity proteins in the mammary epithelium is sufficient to induce premalignant lesions and can accelerate tumor progression in the presence of oncogenes (Zhan et al. 2008; McCaffrey and Macara 2009; Xue et al. 2013; Feigin et al. 2014; Godde et al. 2014; Archibald et al. 2015). It has been proposed that loss of polarity is an early event in carcinogenesis (Huang and Muthuswamy 2010; Huebner et al. 2014). Indeed, ErbB2 expression or depletion of polarity protein Scrib is capable of disrupting cell polarity prior to hyperplasia in organoids or in vivo in mice (Aranda et al. 2006; Godde et al. 2014; Huebner et al. 2014). However, whether loss of cell polarity at the earliest stages of carcinoma development is widespread in human disease has not been established.

The organization of mammary ducts is characterized by the presence of a central lumen formed by a layer of epithelial cells that is surrounded by a basal myoepithelial cell layer, and loss of the luminal space during cancer progression is frequently referred to as luminal filling (Taraseviciute et al. 2010; Leung and Brugge 2012; Venugopalan et al. 2014). One model proposes that luminal filling arises from cells shedding or migrating into the lumen to create solid structures (Taraseviciute et al. 2010; Leung and Brugge 2012). This depends on cells overcoming anoikis and stimulating proliferation inside the lumen (Danes et al. 2008; Leung and Brugge 2012; Pradeep et al. 2012). More recently, it was demonstrated in mouse models of breast cancer that cell divisions oriented perpendicular to the plane of the duct can contribute to stratification of luminal epithelial cells in the absence of cell shedding or migration into the lumen (i.e., luminal filling); however, stratification is not sufficient to generate solid ducts (Godde et al. 2014; Huebner and Ewald 2014; Shore et al. 2016). Therefore, there is controversy regarding the mechanisms that contribute to the generation of solid ducts in experimental models, and how this occurs in human patients is not understood.

Tension at the apical membrane works to maintain lumen shape and size by modulating the actin cytoskeleton through RhoA family GTPases, thus controlling tissue integrity and morphogenesis (Martin and Goldstein 2014). Myosin II associates with actin filaments and contracts following phosphorylation of its light chain by Rho-associated protein kinase (ROCK), which itself is activated by RhoA in its active GTP-bound state (Martin and Goldstein 2014). RhoA activity is regulated at the apical membrane through guanine nucleotide exchange factors (GEFs) and GTPase-activating proteins (GAPs), which enable an appropriate balance of RhoA in its inactive GDP-bound and active GTP-bound states. Importantly, altered RhoA activity has been associated with both anti-tumorigenic and protumorigenic functions, indicating that it behaves as an oncogene or tumor suppressor in different contexts (Zandvakili et al. 2017).

Breast cancer is a heterogeneous disease and is the most common malignancy affecting women worldwide. Although breast cancer-associated mortality has reduced, incidence is increasing (Global Burden of Disease Cancer Collaboration 2015). Genetic, epidemiological, and histo- logical studies indicate that ductal breast cancers can develop through preinvasive stages that include flat epithelial atypia (FEA), atypical ductal hyperplasia (ADH), and ductal carcinoma in situ (DCIS), which are risk factors for development of invasive ductal carcinoma (IDC) (Ellis 2010; Lopez-Garcia et al. 2010; Sgroi 2010; Sinn et al. 2010; Bombonati and Sgroi 2011). However, not all lesions will lead to invasive disease, and it is currently not possible to predict which are most likely to progress (Sgroi 2010), highlighting the need for a better understanding of the mechanisms involved in early breast cancer progression.

Based on the presence or absence of hormone receptors (HRs) to estrogen and progesterone and the human epidermal growth factor receptor 2 (HER2), breast cancers are broadly classified into subtypes that exhibit diverse phenotypes and clinical outcomes (Kos and Dabbs 2016). HR-positive $\left(\mathrm{HR}^{+}\right)$luminal-type carcinomas represent the majority of breast cancers $(60 \%-70 \%)$, and evidence supports that different subtypes are already evident at the DCIS stage, indicating that drivers of different subtypes may act prior to development of invasive disease (Hannemann et al. 2006; Allred et al. 2008; Lopez-Garcia et al. 2010; Tang et al. 2016). Although $\mathrm{HR}^{+}$cancers generally have a better prognosis than other subtypes in the short term, survival declines over $10 \mathrm{yr}$ and, due to the high prevalence of this subtype, accounts for a majority of breast cancer-related deaths (Haque et al. 2012). The cellular and molecular mechanism by which carcinomas develop in the breast and other epithelia remain poorly understood, which impedes progress to prevent cancer development.

Using human breast biopsy samples as well as in vivo mouse models and organotypic cultures, we report diverse mechanisms that contribute to loss of cell and tissue polarity in the development of carcinoma. We identified that a major mechanism observed in generating solid ducts involved progressive loss of cell polarity through asymmetric cell divisions and the collapse of lumina through reduced RhoA/myosin II activity at the apical membrane. In contrast, early loss of apical cell polarity and shedding of cells into the lumen were rarely observed in $\mathrm{HR}^{+}$breast lesions. Finally, we report that loss of tissue organization and cell polarity were reversible upon removal of oncogene stimulation. Together, these results highlight the dynamics of epithelial remodeling during the development of carcinoma and reveal a previously unappreciated mechanism involving progressive polarity loss and luminal collapse as key characteristics.

\section{Results}

\section{Diverse polarity phenotypes are present in DCIS}

To investigate apical-basal membrane polarity in DCIS, we examined $49 \mathrm{HR}^{+}$DCIS lesions from breast biopsies by immunostaining for Par6 and E-cadherin to mark the apical and basolateral membranes, respectively (Fig. 1; Supplemental Fig. S1). We characterized apical-basal membrane polarity, referred to here as apical-basal 
A
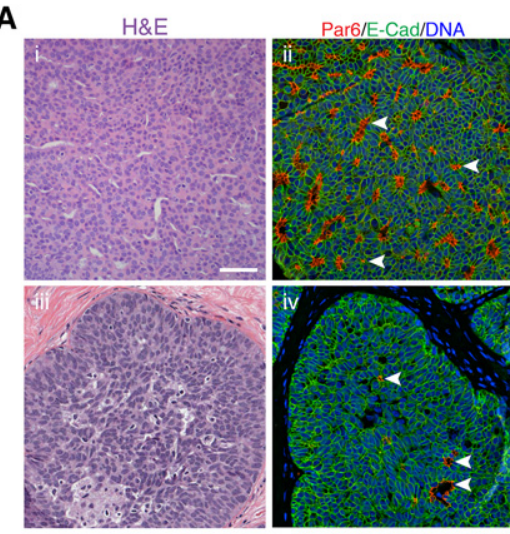

B $_{30}$

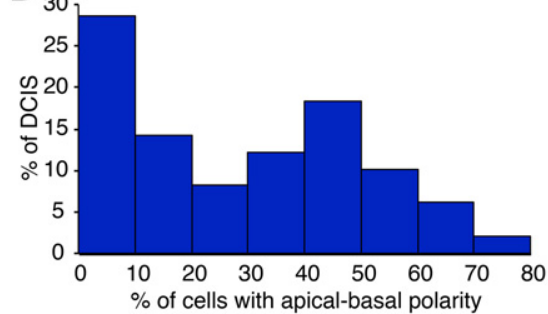

D

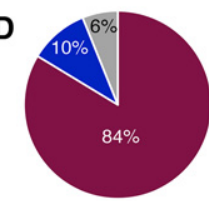

Non-polarized
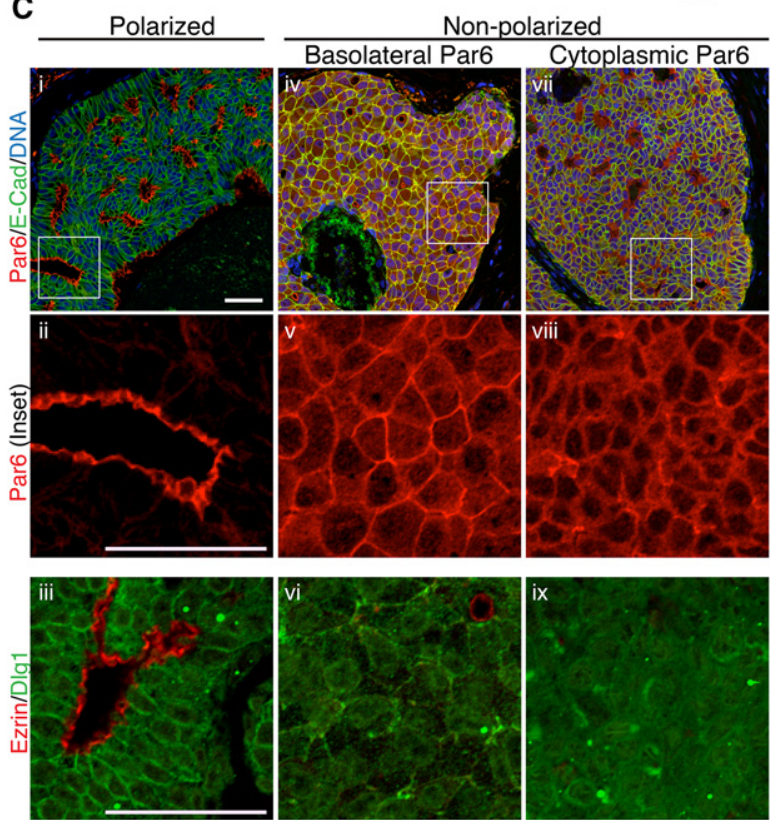

Polarized Par6

Basolateral Par6

Cytoplasmic Par6

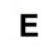

E Basolateral Par6

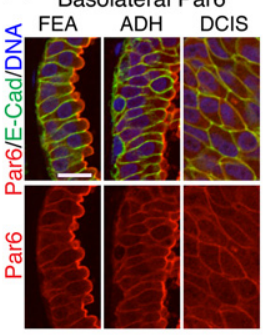

Cytoplasmic Par6

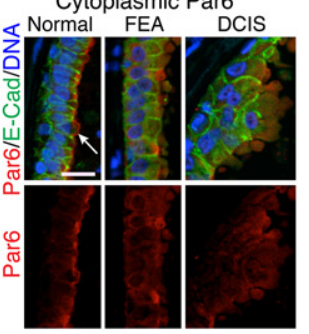

Figure 1. Diversity of apical-basal polarity in DCIS. (A) Histological sections (panels i,iii) and immunofluorescence staining (panels ii, iv) of adjacent sections of DCIS showing examples of apical-basal polarity in DCIS. Arrowheads show examples of cells with polarized Par6. (B) Histogram of the distribution of DCIS with varying frequencies of cells with apical-basal polarity. $n=49$. (C) Immunofluorescence staining of DCIS showing polarized apical Par6 (panels i,ii), basolateral Par6 (panels $i v, v$ ), and cytoplasmic Par6 (panels vii, viii). Panels iii, vi, and ix show immunofluorescence of serial sections stained for Ezrin and Dlg1. $(D)$ Frequency of Par6 phenotypes observed in DCIS. (E) Immunofluorescence of Par6 and E-cadherin on individual patient samples containing DCIS and earlier stages. The arrow shows polarized Par6 in adjacent normal tissue. Bars: $A, C, 50 \mu \mathrm{m}, E, 20 \mu \mathrm{m}$.

polarity for simplicity, as segregation of apical and basolateral plasma membrane domains based on established apical (Par6 and Ezrin) and basolateral (E-cadherin and Dlg1) markers. In most of the DCIS that we analyzed (47 of 49), we observed populations of cells that exhibited apical-basal polarity (range $0.3 \%-80 \%$ ) (Fig. 1A,B). The presence of cells with apical-basal polarity in DCIS reveals that complete loss of polarity is not necessarily an early event during development of carcinoma, indicating that previously unappreciated mechanisms exist to disrupt cell and tissue organization to generate solid ducts.

Less frequently, we also observed additional Par6-staining patterns that were uniform in all cells of the lesion, including Par6 colocalizing with the basolateral marker Ecadherin on membranes (three of 49) and Par6 highly expressed in the cytoplasm (five of 49) (Fig. 1C,D). As expected, an inverse correlation existed between lesions with membrane or cytoplasmic Par6 and the proportion of polarized cells (membrane: Pearson's $\rho=-0.39, P=0.006$; cytoplasmic: Spearman's $\rho=-0.45, P=0.001)$. To determine whether these staining patterns were specific to Par6 or were shared by other apical polarity proteins, we immu- nostained for Ezrin, a subapical protein that associates with the actin cytoskeleton. In samples with polarized Par6, Ezrin was also detected in a polarized pattern, indicating that apical-basal membrane polarity is present in these cells. However, in cells with basolateral Par6, Ezrin was not detected in the basolateral membrane, indicating specificity of mislocalized apical proteins (Fig. 1C). Ezrin was also not detected in cells from DCIS with cytoplasmic Par6 (Fig. 1C), indicating that these cells have lost general apical membrane identity, not just Par6. Since the apical Par polarity complex is known to cross-regulate basolateral polarity proteins, we examined the localization of Dlg1 in DCIS within lesions with different Par6 phenotypes. In DCIS with polarized Par6, Dlg1 was expressed on the basolateral membrane, as expected. In contrast, Dlg1 showed weak and fragmented localization to the plasma membrane or was cytoplasmic in samples with basolateral or cytoplasmic Par6, indicating that apicalbasal membrane polarity is disrupted in these lesions (Fig. 1C,D). To understand whether Par6 localization is altered in other preinvasive lesions, we immunostained samples that contained less advanced stages adjacent to 
DCIS. We observed that in cases with basolateral or cytoplasmic Par6 in DCIS, Par6 mislocalization was also detectable in the FEA stage (Fig. 1E). Similarly, in DCIS with polarized Par6, less advanced lesions also retained apical-basal membrane polarity (Fig. 2A-C). Therefore, most DCIS examined retained a population of cells with apical-basal membrane polarity even when tissue organization was disrupted and ducts appeared solid by standard histological analysis.

\section{Apical-basal polarity is progressively lost during breast carcinoma development}

Solid ducts are a feature of carcinoma progression, and it has been proposed that depolarized cells can shed into the lumen to generate solids ducts (Danes et al. 2008; Leung and Brugge 2012; Pradeep et al. 2012). We examined 114 preinvasive lesions and observed only two that showed epithelial cells populating the luminal cavity (Supplemental Fig. S2A). Interestingly, in both examples, the ducts also had disrupted apical membrane identity, indicating that early loss of apical-basal polarity is a rare event but may be associated with cells entering the lumen. Our finding that the majority of DCIS examined was heterogeneous for cells with and without apical-basal polarity prompted us to examine the apical-basal polarity status in a spectrum of preinvasive human breast lesions, including FEA, ADH, and DCIS as well as invasive carcinoma. FEA are enlarged hyperplastic ducts with one to several layers of abnormal cells but lack architectural alterations such as cribriform patterns or solid ducts that are present in $\mathrm{ADH}$ and DCIS (Fig. 2). We identified samples that contained DCIS with adjacent FEA or $\mathrm{ADH}$ but with no invasive carcinoma, DCIS adjacent to invasive carcinoma, and IDC and scored the proportion of cells exhibiting apical-basal membrane polarity in each type of lesion (Fig. 2B,C). Whereas we observed $~ 80 \%$ of epithelial cells exhibiting apical-basal polarity in FEA, this was reduced to $\sim 60 \%$ in $\mathrm{ADH}$. In FEA and $\mathrm{ADH}$, nonpolarized cells were positioned basally to polarized cells as part of stratified regions surrounding round luminal structures (Fig. 2B,C; Supplemental Fig. S2B). DCIS lesions had smaller regions of polarized cells than seen in $\mathrm{ADH}$. In $\mathrm{ADH}$, cells with apical-basal polarity surrounded well-defined round lumina; however, in DCIS, the lumina were often collapsed, showing thin luminal spaces, or were present as an apical patch with no intercellular space detected (Fig. 2D,E). Although elements of apical-basal polarity persisted in DCIS without
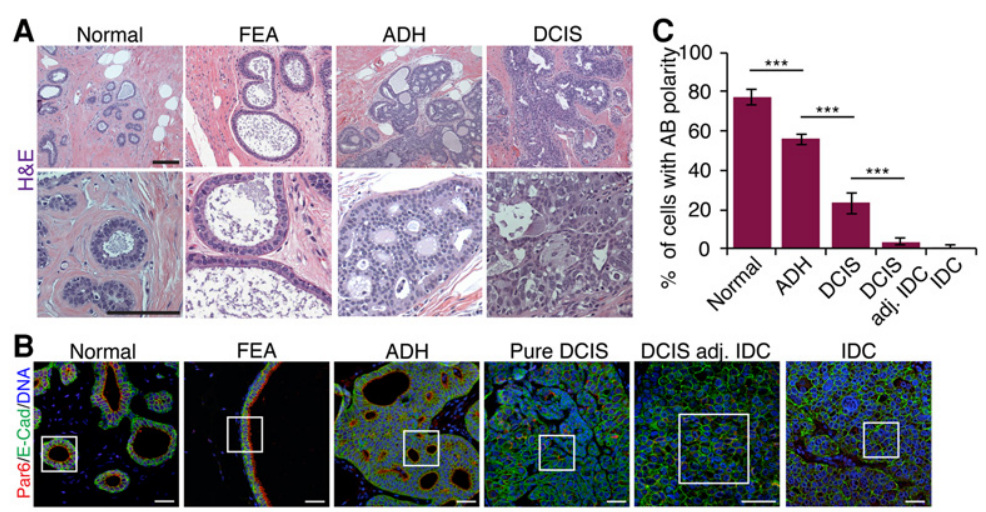
FEA

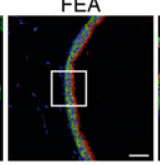
$\mathrm{ADH}$ Pure DCIS
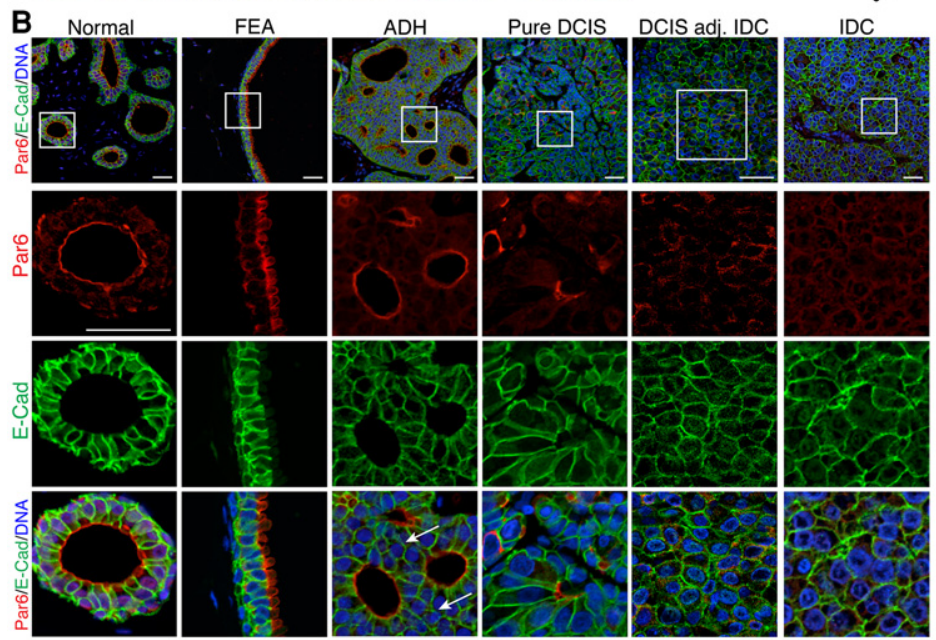

D
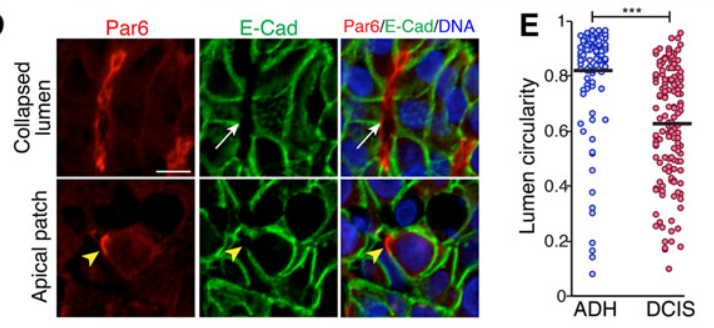

Figure 2. Apical-basal polarity is progressively lost in preinvasive breast lesions. (A) Histological sections from biopsy material containing normal, FEA, ADH, and DCIS. (B) Fluorescence images of tissue sections immunostained for Par6 (red) and E-Cadherin (green) at the indicated stages, including DCIS adjacent to invasive carcinoma (DCIS adj. IDC) and IDC. Arrows show examples of cells lacking apical polarity. $(C)$ Quantification of the percentage of cells that exhibit apical polarity at the indicated stages of progression. At least six image fields from five subjects were analyzed for each. Error bars indicate SEM. $(D)$ Representative fluorescence images from DCIS showing collapsed lumen (white arrow) and apical patch (yellow arrowhead). (E) Quantification of lumen shape (circularity) between ADH and DCIS. Four subjects with both $\mathrm{ADH}$ and DCIS were used to measure 114 lumina for $\mathrm{ADH}$ and 153 lumina for DCIS. Black bars represent the mean. $\left(^{* * *}\right) P<0.001$. Bars: $A, 100 \mu \mathrm{m} ; B, 50 \mu \mathrm{m} ; D, 20 \mu \mathrm{m}$. 
invasive disease, we detected very few cells with apicalbasal polarity in invasive carcinoma or DCIS adjacent to invasive carcinoma (Fig. 2B,C). Therefore, we propose that loss of apical-basal membrane polarity occurs progressively during breast carcinoma development. Furthermore, solid ducts rarely result from cells shed into the lumen, but rather the lumen collapses following stratification of the epithelium.

\section{Polarized cells collectively penetrate the lumen to generate secondary lumina}

Since cell shedding into the lumen is an uncommon event in luminal breast lesions, we next explored the process by which a duct with a single large lumen (FEA) may generate stratified ducts with numerous smaller lumina (ADH). FEAs are considered precursors to ADHs (Sgroi 2010), and we therefore examined three patient samples where FEA was adjacent to ADH. Of the 16 FEA lesions adjacent to $\mathrm{ADH}$, we observed stratified FEAs with finger-like cords of cells extending into the lumen in four cases $(25 \%)$ and bridges that generate secondary lumina in all ADHs (Supplemental Fig. S3A,B). In all cases, the cords contained cells with apical-basal polarity contacting the lumen (Supplemental Fig. S3B). We did not observe any finger-like projections in FEAs that were not adjacent to ADHs. A distinguishing feature of FEA and $\mathrm{ADH}$ is the absence of complex architecture in FEA (Sgroi 2010); therefore, we consider the presence of finger-like projections as an intermediate between FEA and ADH.

We next examined whether the growth properties of simple or stratified epithelia in FEA were altered. Staining for Ki67 revealed that stratified epithelial were more proliferative (Supplemental Fig. S3C,D), suggesting that the stratified epithelium may represent an active state that is amenable to morphogenesis in preinvasive lesions.

\section{Polarity is progressively lost in a mouse model of breast cancer progression}

To further understand the spatiotemporal events leading to loss of apical-basal polarity, we examined tumors from mice expressing the polyoma virus middle-T antigen (PyMT) under the mouse mammary tumor virus (MMTV) promoter/enhancer (Lin et al. 2003) or as a doxycycline-inducible system with a cross of two mouse strains: the PyMT-IRES-CRE (MIC) and the MMTV-reverse tetracycline transactivator (rtTA) (MTB) (Rao et al. 2014). These mice form multifocal $\mathrm{HR}^{+}$luminal-type tumors with high penetrance $(>75 \%)$ that progress through stages, including hyperplasia, mammary intraductal neoplasia (MIN), and adenocarcinoma, that are morphologically similar to human preinvasive breast lesions (Guy et al. 1992; Lin et al. 2003; Herschkowitz et al. 2007; Rao et al. 2014). To determine whether this model resembles human cancer in regard to progressive loss of apical-basal polarity and tissue organization, we immunostained tissue sections from different stages with a panel of apical-basal polarity markers, including ZO1, Par6, aPKC, Ezrin, Ecadherin, and Dlg1 (Fig. 3A; Supplemental Fig. S4A-C).
In hyperplasia, we observed an increase in the number and size of ducts, suggesting that branching morphogenesis is increased at the earliest steps. These ducts then became multilayered with the appearance of collapsed structures or apical patches at later stages, with eventual complete loss of apical-basal membrane polarity, similar to human breast cancer progression (Supplemental Fig. S5A). Like human progression, the proportion of cells exhibiting polarity was gradually lost and progressed through lumen collapse and apical patches in more advanced lesions (Fig. 3A,B; Supplemental Fig. 5A,B). All apical markers showed similar localization patterns, and we did not observe any mislocalization of the basolateral markers examined, confirming the presence of apical-basal membrane polarity in a subset of cells.

The basement membrane can provide cues to establish or maintain apical-basal polarity and is altered during breast cancer progression (Lee and Streuli 2014). Therefore, to further understand changes in cell polarity and tissue organization, we investigated the basement membrane and myoepithelial cells by immunostaining mouse tumor sections for laminin, a prominent basement membrane marker. Normal ducts and hyperplasia/MIN had robust laminin staining around the outside of ducts (Supplemental Fig. S5C). Although some cells in stratified epithelia appeared to lack contact with laminin, laminin was detected surrounding collapsed lumina, supporting the idea that collapsed lumina maintain elements of a cell polarity program and that the myoepithelial cell layer is not responsible for depositing the laminin in these lesions (Supplemental Fig. S5C,D).

As shown above, the fraction of cells with apical-basal polarity and lumen size is reduced as breast lesions progress. We predicted that nonpolarized cells may have a proliferative advantage, allowing them to outgrow the polarized epithelial population. We immunostained for Ki67 and found that nonpolarized cells had an approximately fourfold increase in the proportion of proliferating cells compared with their polarized counterparts (Supplemental Fig. S5E,F). To determine whether polarized cells may be eliminated by apoptosis, we stained for both cleaved Caspase 3 and TUNEL and quantified the number of positive cells that are contacting the lumen or not. Polarity markers were lost in most apoptotic cells; therefore, the quantification was conducted with respect to their position, whether contacting the lumen or more basally positioned. However, we found that cells contacting the lumen had lower apoptosis compared with more basally positioned cells (Supplemental Fig. S5G,H), suggesting that polarized cells are unlikely to be eliminated through cell death during carcinoma development.

To further examine key steps in breast carcinoma progression, we established three-dimensional (3D) organotypic cultures from mammary epithelial cells from MMTV-rtTA-PyMT mice, which drive PyMT oncogene expression through a doxycycline-dependent inducible promoter (Rao et al. 2014). Cells from noninduced mice were cultured to establish polarized structures with lumina, and then doxycycline was added to the culture 


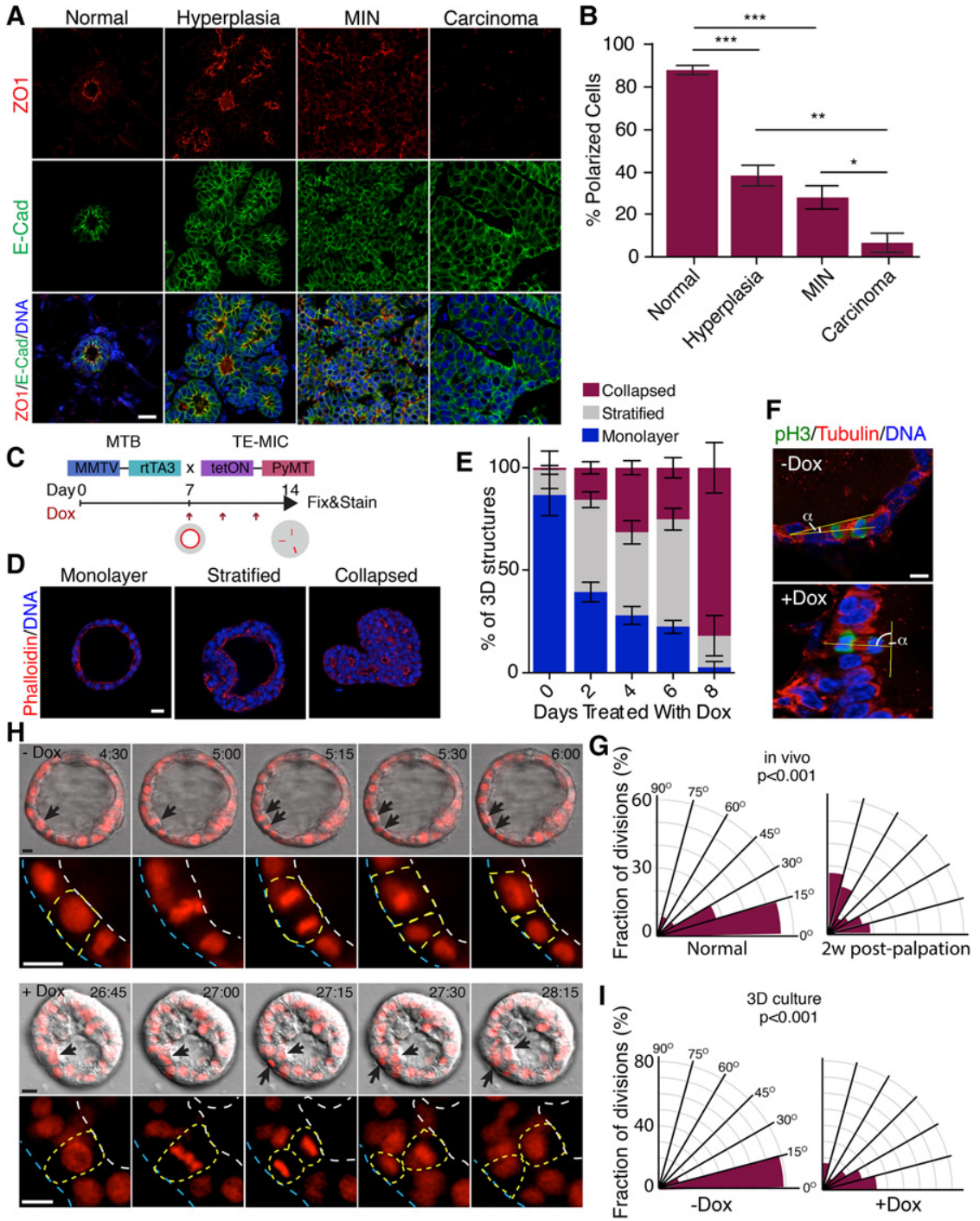

Figure 3. Progressive polarity loss and luminal collapse during mammary tumor progression in mice. (A) Images of normal and tumor tissue from PyMT mice immunostained for ZO1 (red) and E-Cadherin (green). (B) Quantification of polarized and nonpolarized cells at the indicated stages during mammary tumor progression. (C) Schematic diagram of an experimental system for three-dimensional (3D) organotypic cultures. $(D)$ Confocal images of organotypic cultures stained with phalloidin to mark the apical membrane following induction of PyMT. (E) Quantification of the indicated phenotypes at different time points following doxycycline-induced PyMT expression. Fifty structures from three mice were counted. $(F)$ Images of tissue samples immunostained for a-Tubulin (red), phosphoHistone-H3 (green), and DNA (blue). $(G)$ Quantification of the cell division angle relative to the plane of the lumen. $n=11$ normal; $n=98$ hyperplastic lesions. $(H)$ Image series from time-lapse microscopy showing cell division in 3D organotypic cultures from MIC-PyMT cultures induced or not with doxycycline. Arrows show dividing cells. White dotted lines mark the apical surface, blue dotted lines mark the basal surface, and yellow dotted lines outline cells during division. (I) Quantification of the cell division angle relative to the plane of the lumen in organotypic cultures from MIC-PyMT mice. Cell divisions $(n=24)$ from 52 organotypic cultures were examined. Bars: $A, 20 \mu \mathrm{m}, D, F, H, 10 \mu \mathrm{m}$. Error bars represent SEM. medium to induce PyMT expression (Fig. 3C). Cells were fixed every $2 \mathrm{~d}$, and the lumina were visualized by staining F-actin with phalloidin. Before addition of doxycycline, $\sim 90 \%$ of organotypic structures exhibited a single large primary lumen (Fig. 3D,E). After $2 \mathrm{~d}, \sim 50 \%$ of organotypic cultures were multilayered but still contained a large primary lumen. Between 6 and $8 \mathrm{~d}$, organotypic cultures dramatically shifted phenotype, and $\sim 90 \%$ existed as multilayered structures with collapsed lumina. Importantly, the stages from 3D culture were morphologically similar to tumors in vivo (i.e., stratification and luminal collapse). Although the lumina appeared as multiple microlumina in confocal image slices, $3 \mathrm{D}$ reconstructions from $z$-stacks confirmed that they are mostly connected and convoluted collapsed lumina (Supplemental Fig. $\mathrm{S} 5 \mathrm{H})$. Although tumors from PyMT mice do not go through stages that resemble human FEA or $\mathrm{ADH}$, they do form stratified epithelial ducts, progressively lose cell polarity, and undergo luminal collapse, key elements observed in the majority of human $\mathrm{HR}^{+}$breast lesions examined.

\section{Asymmetric divisions contribute to stratification}

Our data from human and mouse lesions indicate that establishing stratified ducts may be one of the earliest events in early breast cancer progression. Oriented cell divisions are essential to maintain normal epithelial architecture in many tissues, including the mammary gland, and cell divisions perpendicular to the duct can generate multilayered mammary ducts (Godde et al. 2014; Huebner et al. 2014). We therefore examined whether misoriented divisions occurred in vivo in the PyMT model. We immunostained tissue sections for phospho-Ser10-Histone to label mitotic cells and for a-tubulin to label the mitotic spindle in normal and PyMT lesions (Fig. 3F). In normal ducts, cells predominantly divided within the plane of the duct $\left(0^{\circ}-30^{\circ}\right)$, whereas there were rare oblique $\left(30^{\circ}-\right.$ $\left.60^{\circ}\right)$ or perpendicular $\left(60^{\circ}-90^{\circ}\right)$ divisions (Fig. 3F,G). Interestingly, rare out-of-plane divisions in normal ducts have also been observed previously (Godde et al. 2014). In contrast, cells in PyMT-expressing ducts that were not yet stratified divided in random orientations (Fig. 3F, 
G), supporting that misoriented cell divisions contribute to the formation of stratified ducts and disrupt epithelial organization.

To further examine whether the products of misoriented divisions were sufficient to establish and maintain multilayered ducts, we labeled cell nuclei of mammary cells with mCherry-tagged Histone-H2B and tracked the fate of cell divisions in 3D cultures (Fig. 3H,I; Supplemental Fig. S6A). In noninduced cultures, cells predominantly divided within $30^{\circ}$ of the plane of the lumen, with rare oblique divisions occurring at an angle of $30^{\circ}-60^{\circ}$. However, following oblique divisions, the cells resolved to maintain a simple epithelial organization (Supplemental Fig. S6A). Doxycycline induction of PyMT resulted in cells more frequently dividing in oblique or perpendicular orientations relative to the lumen, which resulted in one daughter cell retaining an apical position and one adopting a basal position (Fig. 3H,I; Supplemental Fig. S6B). Importantly, we tracked cell fates for several hours after division and found that basal cells were permanently excluded from an apical position. Moreover, we did not observe any stratification arising from cell rearrangement or migration alone.

Loss of cell polarity can disrupt cell division orientation, and previous reports indicate that loss of apical-basal polarity occurs prior to stratification and hyperplasia in mouse models of breast cancer (Godde et al. 2014; Huebner et al. 2014). To determine whether polarity was disrupted prior to misoriented cell divisions in PyMT mammary glands, we examined Par6 staining in dividing cells in hyperplasia. We found that Par6 was present at the apical membrane, indicating that apical-basal polarity is intact during randomly oriented cell divisions in this model (Supplemental Fig. S6C). Therefore, this reveals that nonpolarized cells in the stratified epithelium arise from asymmetric divisions in which one cell retains contact with the lumen and apical identity and the other attains a basal position and lacks apical identity.

\section{Reduced RhoA activity is associated with luminal collapse}

Since the actomyosin cytoskeleton controls apical integrity and lumen size (Martin and Goldstein 2014), we investigated its putative role in luminal collapse. Myosin II is activated through phosphorylation by Rho kinase (ROCK1/2) to control tight junction and apical integrity (Zhan et al. 2008). To investigate whether myosin II activity was altered in collapsed lumina, we immunostained for phospho-myosin II in tissue sections from induced or noninduced PyMT mice. Whereas normal ducts exhibit robust phospho-myosin that localized to the apical membrane, phospho-myosin was less intense and diffuse in doxycycline-induced glands (Fig. 4A,B). To confirm and extend this observation, we also examined RhoA-GTP by immunostaining tissue sections with an active statespecific antibody (Kuipers et al. 2014). Indeed, we also observed that RhoA-GTP was reduced in ducts induced with PyMT, and closer examination indicated that RhoA-GTP was low in early transformed ducts that were not yet strat- ified or collapsed, indicating that it precedes lumen collapse in this model (Fig. 4C-E). In human premalignant lesions, $\mathrm{ADH}$ exhibits well-developed secondary lumina, but lumina were frequently collapsed in DCIS. We therefore evaluated samples containing both ADH and DCIS with collapsed lumina to determine whether RhoA-GTP was also reduced in human samples (Fig. 4F,G). Indeed, we observed a reduction in RhoA activity in collapsed lumina, supporting that loss of apical integrity associated with luminal collapse may result from reduced RhoA/myosin II activity.

We next sought to test whether restoring RhoA activity could block luminal collapse. Reduction of RhoA/myosin activity at the apical membrane could be due to either reduced RhoGEF or increased RhoGAP activity. We first investigated whether there was a change in a RhoGEF at the apical membrane in collapsed lumina. One candidate GEF is p114RhoGEF, which is expressed on the apical domain in epithelial cells, is required to maintain apical integrity, and regulates epithelial morphogenesis (Nakajima and Tanoue 2011; Terry et al. 2011). However, immunostaining of tumor sections from PyMT tumors showed strong expression of $\mathrm{p} 114 \mathrm{RhoGEF}$ on both open and collapsed lumina (Supplemental Fig. S7A). The presence of p114RhoGEF on the apical membrane of collapsed lumina suggests that loss of apical GEF activity is unlikely to be responsible for reduced RhoA and myosin II activity in collapsed lumina, although we cannot exclude the possibility that other apical GEFs are altered. Instead, we predicted that altered GAP activity may affect RhoA activity during luminal collapse. p190BRhoGAP (p190B) regulates RhoA during mammary gland morphogenesis, and increased p190B expression was found in poorly differentiated mammary tumors in mice (Chakravarty et al. 2000; VargoGogola et al. 2006). Moreover, p190B haploinsufficiency in the mammary epithelium was reported to inhibit ErbB2-mediated mammary tumor formation (HeckmanStoddard et al. 2009). We therefore investigated whether p190B was altered following doxycycline induction of PyMT. Following doxycycline-induced PyMT expression in mammary glands, we observed that p190B immunostaining was more intense and diffusely distributed throughout cells (Supplemental Fig. S7B). Specificity of the antibody was confirmed by knockdown (Supplemental Fig. S7C,D). Therefore, we investigated whether reducing p190B could block luminal collapse in the 3D organoid model. We identified two independent shRNAs that efficiently reduced p190B in mammary epithelial cells and noted that knockdown alone had little effect on cyst growth and structure. (Supplemental Fig. S7C,D). Upon PyMT induction by adding doxycycline to the culture medium, we noted that p190B knockdown impaired both lumen collapse and the generation of solid organoids (Fig. 5A,B).

Finally, we examined whether depleting p190B could reduce luminal collapse in vivo. We depleted p190B from mammary epithelial cells and performed transplants into the cleared mammary fat pad. After regeneration of the epithelium for $5 \mathrm{wk}$ in the absence of doxycycline, we induced PyMT for an additional 2 wk. We observed that 


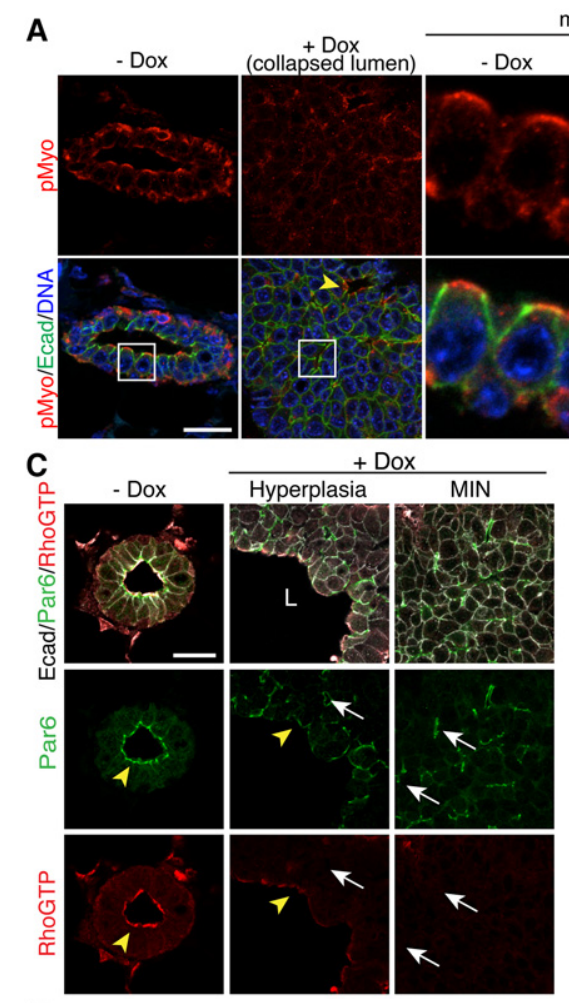

$\mathbf{F}$

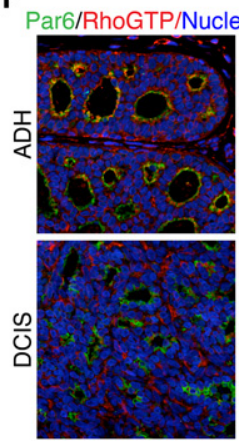

magnified

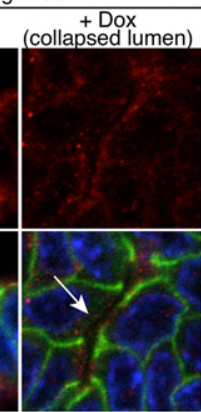

D

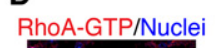

B

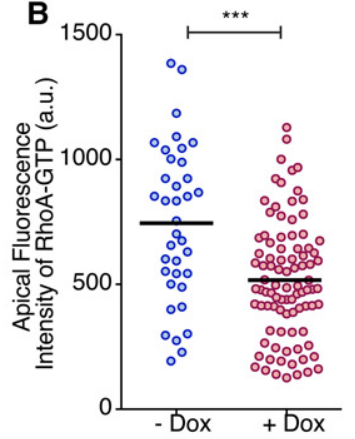

E

Mouse (PyMT)

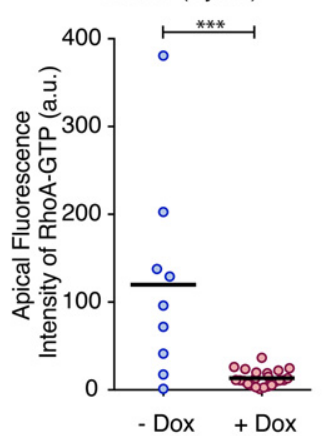

Figure 4. Luminal collapse is associated with disrupted apical integrity. $(A)$ Image of mouse mammary glands with or without doxycycline-induced PyMT expression immunostained for phospho-myosin II (red) and E-Cadherin (green). Yellow arrowheads show open lumina, and white arrows show collapsed lumina. (B) Quantification of phospho-myosin II intensity at the apical membranes. Five tissue sections each were analyzed from three mice. (C) Images of mouse mammary glands with or without doxycycline-induced PyMT expression immunostained for Par6 (red) and active RhoA (RhoGTP; green). Yellow arrowheads show open lumina, and white arrows show collapsed lumina. (D) Image showing RhoA-GTP in open ducts in glands with or without doxycycline-induced PyMT. The bottom panel shows a duct that has not collapsed. (E) Quantification of active RhoA (RhoGTP) at the apical membrane. Three tissue sections each were analyzed from three mice. $(F)$ Images of adjacent $\mathrm{ADH}$ and DCIS immunostained for Par6 (green) and RhoA-GTP (red). (G) Quantification of apical intensity of RhoA-GTP in ADH $\mid n=$ 29 lumina) and DCIS ( $n=52$ lumina) from four human subjects. Bars: $A, C, 20 \mu \mathrm{m}_{i} E$, $50 \mu \mathrm{m}$.

p190B-depleted glands retained a more organized structure with significantly more lumina maintained compared with glands expressing PyMT with control shRNA (Fig. 5C,D). Importantly, we confirmed that RhoA-GTP was retained in ducts depleted of p190B (Fig. 5C). Depletion of p190B in vivo did not significantly affect the proliferation rate or orientation of cell division (Supplemental Fig. S7E, F), suggesting that reduced RhoA activity may not be required for stratification. Despite the retention of lumina, in some ducts, we observed cells invading basally from ducts in p190B-depleted glands, which was not observed with control shRNA or in parental PyMT tumors or 3D organoid cultures in vitro (Fig. 5A; data not shown). We attribute this to sustained RhoA activity caused by GAP depletion, having multiple effects in vivo, which highlights the tight spatio-temporal control of RhoA during mammary gland development and cancer progression. Nonetheless, our in vitro and in vivo data support that luminal collapse and disrupted tissue organization are at least partially due to reduced RhoA activity.

\section{Loss of apical-basal polarity is reversible}

We observed a series of morphological steps that occur during progression of breast cancers that contribute to loss of apical-basal polarity and generate solid ducts. We next asked whether this process was reversible if the stimulating oncogene was removed. To test this, we induced PyMT mice for $2 \mathrm{wk}$ to generate early stage mammary tumors with multilayered collapsed epithelial ducts and then deinduced for $3 \mathrm{~d}, 1 \mathrm{wk}$, or $4 \mathrm{wk}$ by removing doxycycline from the drinking water (Fig. 6A). In all mice examined, we found that deinduction rapidly reverted to ducts with apical-basal cell polarity and a hollow lumen (Fig. 6B-D). Within $3 \mathrm{~d}$ of doxycycline withdrawal, a ring of cells formed that contained a heterogeneous population of cells with and without apical identity (Fig. 6B,C). However, by 1 wk after deinduction, the proportion of cells exhibiting apical-basal membrane polarity was not significantly different from control normal ducts (Fig. $6 \mathrm{~B}, \mathrm{C})$. Deinduction of oncogenes can result in apoptosis 


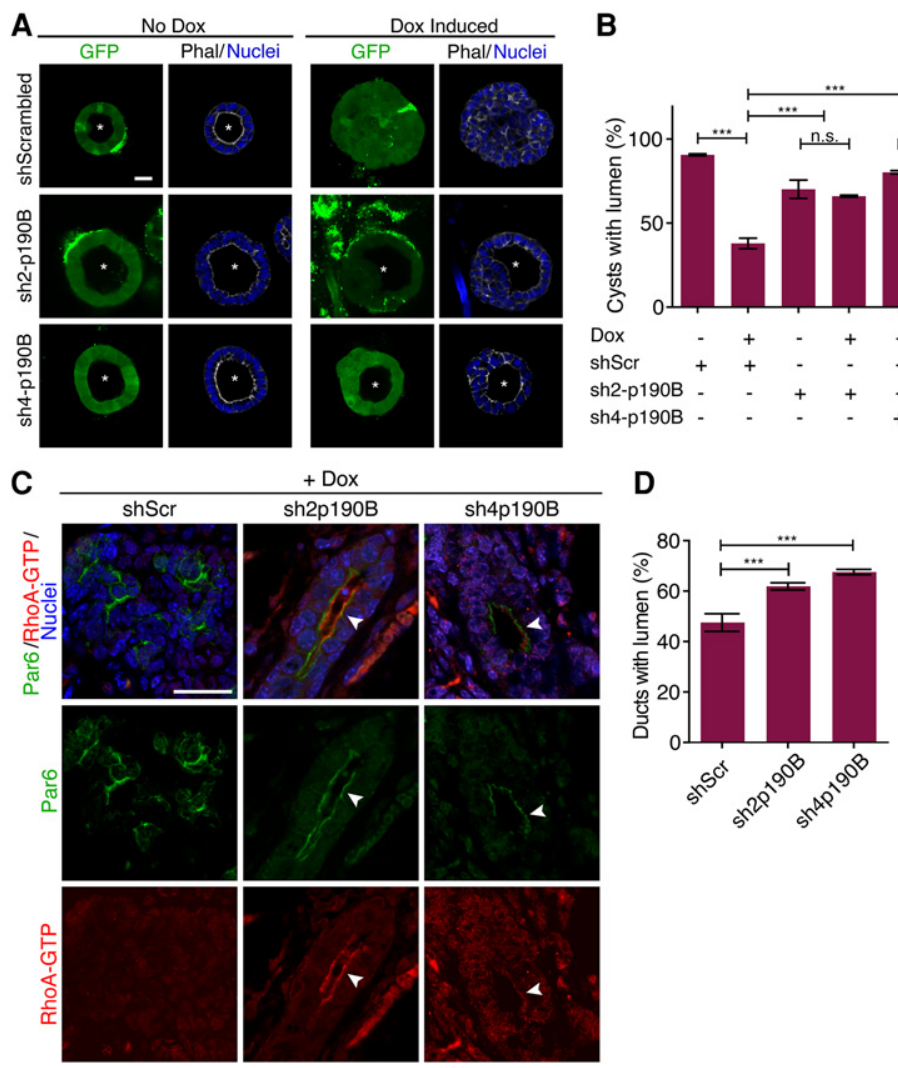

Figure 5. Regulation of RhoA activity regulates lumen collapse. $(A)$ Images of 3D organotypic cultures with or without doxycycline-induced PyMT expression in the presence of scrambled (shScr) or two different p190BRhoGAP shRNAs and immunostained for Par6. GFP marks cells expressing shRNA. Asterisks indicate open lumina. (B) Quantification of lumen phenotypes in cysts. (C) Images of sections of mouse mammary glands with or without induction of PyMT and expression of p190B-RhoGAP shRNA immunostained for Par6 (green) and RhoA-GTP (red). (D) Quantification of ducts with open lumina. Two tissue sections were analyzed from each of four mice. $\left.{ }^{* * *}\right) P<0.001$. Bars, $25 \mu \mathrm{m}$.

and lumen clearing in culture (Jechlinger et al. 2009), and we therefore examined whether apoptosis was also involved in the more complex in vivo environment (Fig. $6 \mathrm{E}, \mathrm{F}$ ). After $3 \mathrm{~d}$ of deinduction, we observed that the cores of the ducts were filled with apoptotic cells. At $1 \mathrm{wk}$ after induction, the cores were mostly free from apoptotic cells but retained debris, which was cleared by 4 wk. Interestingly, during the spike in apoptosis at $3 \mathrm{~d}$ of deinduction, a relatively high proportion of cells in the reforming duct was also apoptotic (Fig. 6E, arrows). Finally, we examined whether RhoA-GTP was restored to the apical membrane of reforming ducts. Although there was a significant increase in the number of cells with apical-basal polarity at $3 \mathrm{~d}$ of deinduction, RhoA was not restored at this time point (Supplemental Fig. S8A,B). At 4 wk of deinduction, RhoA-GTP was significantly higher than in the induced glands; however, it had not returned to levels observed in normal ducts by this time point (Supplemental Fig. S8A,B). We next asked whether more advanced tumors that had lost apical basal polarity were also able to restore polarized epithelial organization. We induced PyMT for 8 wk and then deinduced for an additional 9 wk. Surprisingly, the deinduced glands could restore the number of polarized cells back to $70 \%-80 \%$ (which was slightly lower than in normal ducts) and the number of ducts containing lumina back to normal levels (Fig. 6BD). This demonstrates that epithelial remodeling in mammary tumor progression is dynamic and that disruption of cell and tissue polarity is reversible, at least in this model.

\section{Discussion}

Loss of polarity is a progressive event in luminal breast carcinoma development

The development of breast carcinoma is associated with loss of growth control and disrupted tissue organization in the form of solid ducts that lack a central lumen. To understand loss of polarity in early progression of breast cancers, we examined apical-basal polarity markers in $\mathrm{HR}^{+}$DCIS and made the unexpected discovery that many DCISs contained populations of cells with elements of apical-basal membrane polarity. A closer analysis revealed that polarized cells were organized around multiple small collapsed lumina or apical patches distributed throughout the lesions. This reveals that although tissue organization is largely disrupted in DCIS, individual cells can retain elements of cell polarity. However, these cells are unlikely to be normal and may have other alterations.

Invasive breast cancers are heterogeneous, which has a significant impact on therapeutic response and relapse (Zhang et al. 2017), but heterogeneity in preinvasive lesions is less well characterized. We report that within preinvasive lesions, cellular heterogeneity exists with respect to apical-basal polarity. Since apical-basal membrane polarity is an important regulator of cell behavior, including control of signaling, proliferation, survival, and invasion (Halaoui and McCaffrey 2015), heterogeneity in polarity status may enable altered signaling in polarized and nonpolarized cells that may promote their progression to invasive carcinoma. In support of this, we 


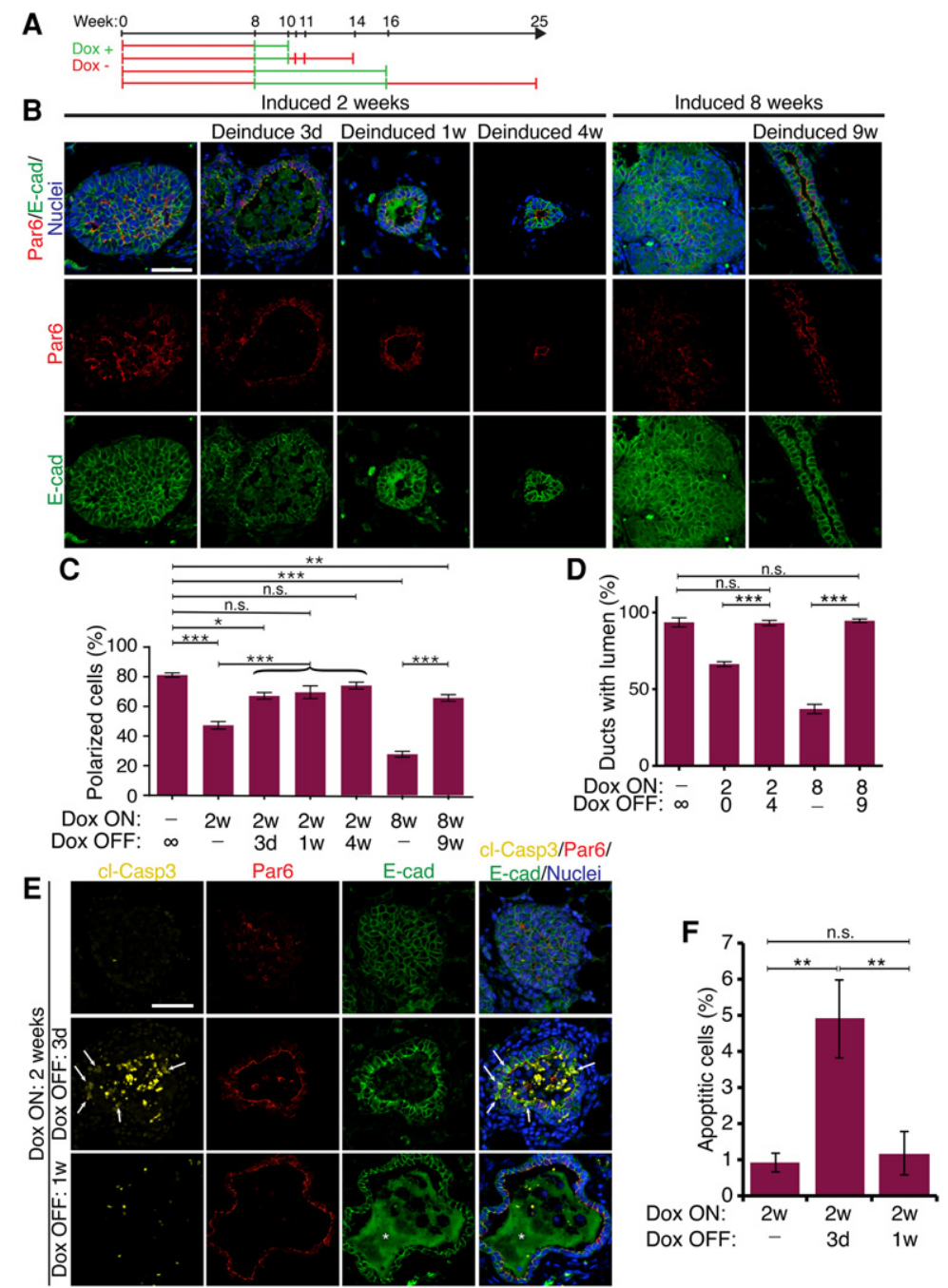

Figure 6. Disruption of tissue organization and cell polarity is reversible. (A) Scheme showing doxycycline treatment schedule for PyMT induction and deinduction. (B) Images of mammary tissue immunostained for Par6 and E-cadherin after doxycycline-mediated induction/deinduction for specified times. $(C)$ Quantification of cells with apical-basal membrane polarity from induction/deinduction cycles. (D) Quantification of ducts with open lumina from induction/deinduction cycles. (E) Images of mammary tissue immunostained for Par6, E-cadherin, and cleaved Caspase 3 after doxycycline-mediated induction/deinduction for the indicated times. The white asterisk indicates fluorescence from debris in the lumen. $(F)$ Quantification of the number of cleaved Caspase-3-positive cells in E-cadherin-positive ductal cells. $\left(^{*}\right) P<0.05 ;\left(^{* *}\right) P<0.01 ;\left(^{* * *}\right) P<0.001$. Bars: $50 \mu \mathrm{m}$. observed that nonpolarized cells were more proliferative than their polarized counterparts. Moreover, invasive breast cancers examined by us here and previously (McCaffrey et al. 2012) were found to be comprised of cells that lack apical-basal polarity. Therefore, disrupted polarity cues may contribute to heterogeneous cell behaviors in the progression of preinvasive breast lesions that may promote development of invasive carcinoma. Moreover, although preinvasive lesions in which some cells retain apical-basal polarity were most common in our data set, we also observed other phenotypes, including basolateral or cytoplasmic Par6 and rare ducts in which apical-basal polarity was lost and cells shed into the lumen. Whether these different phenotypes are associated with different potentials to progress to invasive carcinoma is not known at present and will need to be evaluated in the future.

Luminal filling caused by cells shedding or migrating into the lumen and evading anoikis has been proposed as a mechanism to generate solid lumina (Leung and Brugge 2012; Pradeep et al. 2012). In our data set of preinvasive luminal breast lesions, we observed intraluminal epithelial cells only rarely (two of 114 lesions), indicating that although this process can occur, it is not the primary mechanism that generates solid lesions in DCIS. Instead, we found that luminal collapse of stratified ducts and progressive loss of apical-basal polarity contribute to generate solid ductal structures. The differences in luminal filling versus stratification and luminal collapse may be explained by the presence or absence of tight junctions. Luminal filling has been characterized predominantly in MCF10A cells, which lack tight junctions and apical membrane identity (Fogg et al. 2005; Mailleux et al. 2008). In support of this, the two lesions that we identified with luminal filling had disrupted apical polarity. However, disruption of Scrib or expression of ErbB2 also eliminates tight junctions and cell polarity but does not induce cell shedding into the lumen (Godde et al. 2014; Huebner et al. 2014), suggesting that other factors may also contribute.

The PyMT model is a widely used mouse model of breast cancer that recapitulates signaling features of luminal breast cancer and progresses through preinvasive stages that are histologically and molecularly similar to human breast cancer progression (Maglione et al. 2001; Lin et al. 2003; Herschkowitz et al. 2007; Fluck and Schaffhausen 2009; Rao et al. 2014). Human cancers are thought 
to initiate from alterations in individual cells, and although this initiating event may not be recapitulated in transgenic models that have uniform expression of an oncogene, the PyMT model is a useful tool to study progression because of its high penetrance, short latency, and highly reproducible progression to carcinoma (Guy et al. 1992; Rao et al. 2014). Based on our observations from PyMT mice in vivo and in organotypic cultures, we propose that increased proliferation initially expands the number and size of ducts while maintaining relatively normal tissue organization. The next step involves breaking tissue polarity and creating a stratified epithelium. It was reported previously that mammary stratification is induced by cell divisions oriented perpendicular to the duct plane as an early event in hyperplastic growth in response to oncogene activation or tumor suppressor inactivation (Godde et al. 2014; Huebner et al. 2014). In these models, misorientation of the mitotic spindle was associated with loss of apical-basal polarity either by directly disrupting the polarity machinery or through expression of the ErbB2 oncogene, which can bind to and disrupt the Par polarity complex (Aranda et al. 2006; Godde et al. 2014; Huebner et al. 2014). Our results from PyMT mouse lesions similarly show that perpendicular divisions are the primary driver that generates stratified epithelial structures early during tumor progression. However, one important difference with the PyMT model is that apical-basal membrane polarity is not lost from the luminal cells. Instead, cells undergo an asymmetric cell division to generate a daughter cell that retains an apical position and apical membrane identity and a basally positioned daughter that does not contact the lumen and lacks apical-basal polarity. The contribution of asymmetric divisions that maintain a population of polarized cells and generate nonpolarized cells is consistent with gradual reduction in the proportion of polarized cells that we observed during progression in humans and PyMT mice.

We propose that similar processes may take place during preinvasive breast cancer progression in humans (Fig. 7). Genetic and histological data support a model in which stepwise progression through FEA, $\mathrm{ADH}$, and DCIS is a nonobligate precursor of invasive carcinoma (Sgroi 2010; Sinn et al. 2010). FEAs present as enlarged ducts that are organized as one to several layers. Since cell divisions are rare in human samples, we were unable to identify any cells dividing in FEA to determine whether division orientation is altered in stratified samples. However, perpendicular division can lead to mammary gland stratification in a variety of normal and tumorigenic contexts, as described here and previously (Godde et al. 2014; Huebner and Ewald 2014), and it seems likely that this may also contribute to stratification in humans as well. In our human experimental data set, we observed that stratified FEA retained apical-basal polarity in cells adjacent to the lumen and cells that lack apical-basal polarity in a more basal position, similar to what we observed in PyMT mice and organotypic cultures, raising the possibility that asymmetric divisions generate cells that lack apical-basal polarity in human breast lesions as well. The formation of a stratified epithelium may initiate an acti-

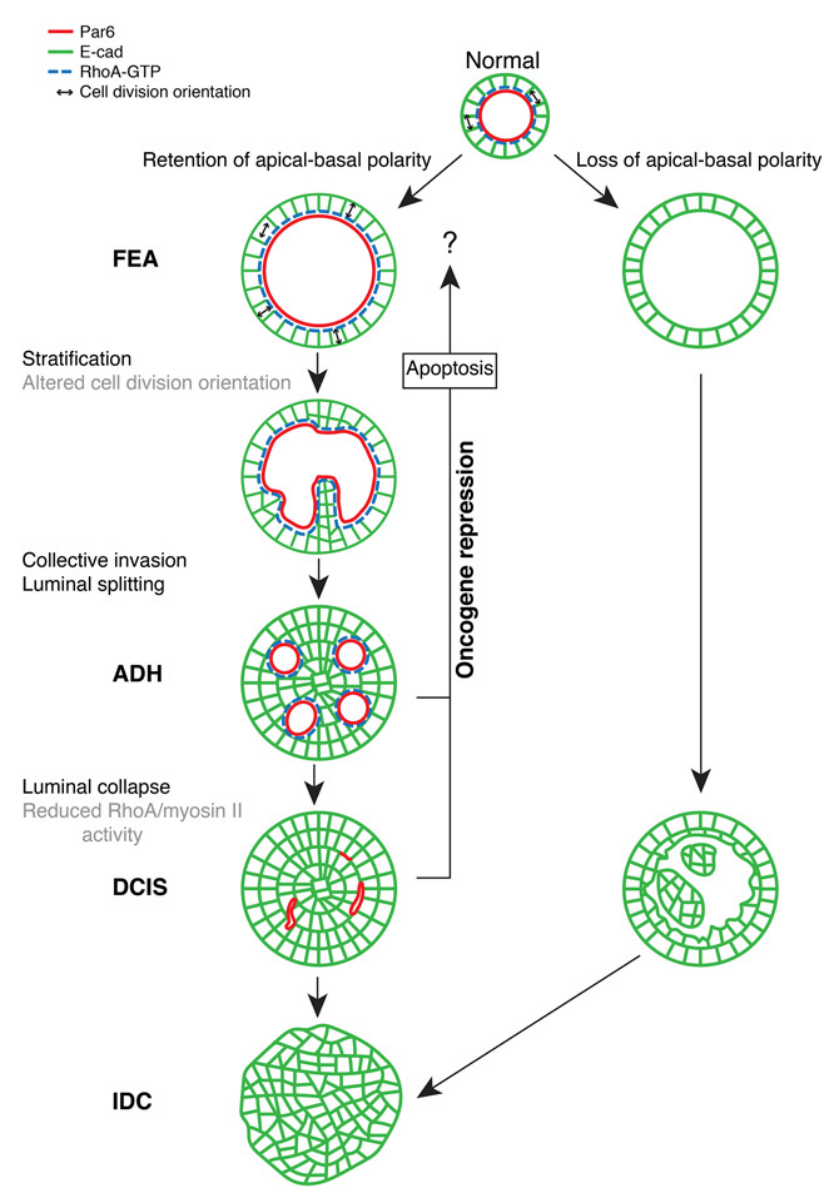

Figure 7. Model for loss of apical polarity and lumen filling in $\mathrm{ER}^{+}$breast cancers. The model shows progressive loss of polarity (left) and early loss of polarity (right). See the Discussion for details.

vated epithelial state that permits dynamic tissue remodeling during progression to later stages. Indeed, the generation of a stratified epithelium is coincident with dynamic cell rearrangements that promote branching morphogenesis and ductal growth during mammary development, and collective migration occurs from stratified epithelial structures in advanced carcinoma (Nguyen-Ngoc et al. 2012; Cheung et al. 2013; Huebner and Ewald 2014). Our observations indicate that stratified epithelia may be involved in tissue remodeling during preinvasive breast cancer progression, since we observed finger-like cords extending from stratified regions that form intraluminal bridges and secondary lumina in $\mathrm{ADH}$, a process that we call lumen splitting (Fig. 7). Importantly, the luminal edges of finger-like projections retain apical identity, which is important to maintain apical-basal polarity in secondary lumina of cribriform structures.

Luminal collapse generates solid duct structures in $\mathrm{HR}^{+}$ carcinoma progression

Importantly, we observed a progressive reduction in the proportion of cells exhibiting apical-basal membrane 
polarity in more advanced human lesions, suggesting that, similar to the PyMT model, human preinvasive cancers develop with progressive loss of apical-basal polarity. Moreover, we also observed collapsed lumina in more advanced lesions in both the PyMT model and human DCIS. Lumen collapse appears to depend on reduced RhoA/myosin activity at the apical membrane, since both were reduced at the apical membrane following PyMT induction, and restoring RhoA at the apical membrane by depleting p190B-RhoGAP prevented luminal collapse. Intriguingly, loss of RhoA/myosin was observed prior to lumen collapse, indicating that it is permissive to collapse but not sufficient. RhoA has been shown to have both oncogenic and tumor-suppressive functions (Zandvakili et al. 2017). Our data support a mechanism by which RhoA has tumor-suppressive activity in the early stages of breast cancer progression to maintain lumen architecture of ducts. Consistent with this, a decrease in the progression of preneoplastic lesions in MMTV-Neu mice was also observed with p190B haploinsufficiency (Heckman-Stoddard et al. 2009). In some p190B-depleted ducts, we observed increased invasion of cells, likely due to increased global RhoA activity. This highlights the significance of spatio-temporal regulation of RhoA that contributes to tumor-suppressive and oncogenic functions during cancer development. In our human data set, we found that collapsed lumina were observed only in DCIS. Interestingly, we observed high apical RhoA at open lumina in $\mathrm{ADH}$, but it was significantly reduced in collapsed lumina of DCIS, indicating that reduced apical RhoA may be relevant in the development of DCIS in humans.

Apical constriction is a force-generating mechanism that uses actomyosin contractility to reduce lumen size (Martin and Goldstein 2014). Our data showing reduced apical RhoA/myosin II argue against apical constriction as a force to generate the collapsed lumen phenotype in preinvasive breast lesions. The force required to collapse lumina is unknown at present, but we hypothesize that it could result from forces within the stratified epithelium or increased proliferation of nonpolarized cells, which would cooperate with reduced apical integrity to collapse lumina. In support of this concept, tissue forces in the Drosophila wing pouch were found to arise from differential cell proliferation rates rather than apical constriction (Mao et al. 2013).

\section{Loss of apical-basal polarity and tissue organization} is reversed upon oncogene removal

Deinduction of oncogene expression in PyMT and other transgenic mouse models results in tumor reversion in vivo, but it is not known whether cell polarity and duct organization are restored (Liu et al. 2011; Rao et al. 2014). Remarkably, we found that disruption of tissue and cell polarity was reversible and that deinducing PyMT resulted in polarized ducts even after advanced carcinomas that mostly lacked cell polarity were established. This suggests that polarity was not irreversibly suppressed in these lesions but that cells were transiently impaired in their abil- ity to maintain cell polarity. Re-establishing duct organization with a lumen was rapid and involved apoptosis of internal cells to clear the luminal space. Similar clearing of a lumen through apoptosis was observed for other oncogenes in 3D culture (Jechlinger et al. 2009), indicating that there may be a common underlying mechanism. At present, we do not understand how some cells are protected from apoptosis, but their position at the outside suggests that interactions with the extracellular matrix may provide survival cues. However, we observed that many cells adjacent to the stroma in the emerging ductal epithelium were also apoptotic, suggesting that other factors may be required for survival and that additional epithelial remodeling may contribute. Interestingly, RhoA was not present at the lumina of newly emerging ducts, indicating that it is not involved in reforming the lumen, but we cannot exclude that it may have other functions in epithelial remodeling during this phase.

Our finding that preinvasive stages are reversible raises the possibility that early breast lesions may be manipulated pharmacologically to restore or maintain organized ducts to prevent progression of premalignant lesions or recurrence of breast cancer in the future.

\section{Materials and methods \\ Human breast cancer tissue}

Biopsy samples containing normal tissue, FEA, ADH, DCIS, and IDC were analyzed by a certified pathologist who is director of breast pathology at the McGill University Health Centre using standard histological scoring parameters. Tissue sections adjacent to those for histological assessment were immunostained for polarity markers and other proteins described below. Luminal lesions were confirmed by the presence of ERa and PR, as assessed by immunohistochemistry. All procedures involving human patient tissues were approved by the McGill Institutional Review Board (IRB; A03-M24-15A).

\section{Mouse models}

All mice used were in an FVB background. Tumors from MMTVPyMT mice were collected at 4 and 8 wk after the tumor was first detected for hyperplasia/MIN and carcinoma, respectively. For mice with inducible expression of PyMT, MIC mice were crossed with MTB mice (Rao et al. 2014), and pups heterozygous for both genes were selected. Mice $\geq 8$ wk of age were given $2 \mathrm{mg} / \mathrm{mL}$ doxycycline in their water and sacrificed after 2 or 8 wk of induction for hyperplasia/MIN and carcinoma, respectively. All procedures involving animals were approved by the McGill University Animal Care Committee.

\section{Immunostaining and imaging}

Human breast samples were fixed in formalin and cut at 3- $\mu \mathrm{m}$ thickness. Mouse mammary tissue was fixed in either 4\% PFA or Carnoy's fixative, as described previously (McCaffrey et al. 2012), and cut at 8- $\mu \mathrm{m}$ thickness. Deparaffinization and immunostaining were performed as described previously (McCaffrey et al. 2012). The primary antibodies used were as follows: cleaved-Caspase-3 (1/300; Cell Signaling, 9661), Dlg1 (1/100; US Biologicals, S0095-30), E-Cadherin (1/500; BD Transduction, 610181), Ezrin (1/300; Cell Signaling, 3145), Ki67 (1/300; Abcam, 
ab15580), Par3 (1/250; Millipore, 07-330), Par6B (1/300, Santa Cruz Biotechnology, sc-67393), phalloidin (1/100; Invitrogen, A34055), phospho-Histone-H3 (1/250; Cell Signaling, 9701), aPKCı (1/500; BD Transduction, 610175), phospho-myosin light chain II (Cell Signaling, 3671), pan-laminin (Abcam, ab14055), RhoA-GTP (NewEast Biosciences, 26904), p190-B(BD Transduction, 611613), $\alpha$-Tubulin (1/250; Millipore, MAB1864), and $\alpha$-Tubulin (1/500; Sigma, T9026). Secondary antibodies (Alexa fluor 488,555 , and 647) were used at a 1/750 dilution for $1 \mathrm{~h}$ at room temperature. For TUNEL staining, tissue sections were prepared and stained using the TumorTACs kit according to the manufacturer's instructions (Trevigen), except 3,3'-diaminobenzidine (DAB) chromogen was replaced with Alexa fluor 555 Tyramide Signal Amplification (ThermoFisher) for fluorescence detection. Confocal imaging was performed using a LSM700 from Zeiss with $20 \times / 0.8$ NA or $40 \times / 1.4$ NA lenses.

Image processing of brightness and contrast was performed in ImageJ and was applied uniformly to the whole image.

\section{Tissue section measurements}

To count the number of polarized cells in human and the mouse tissues, sections were taken from normal and tumor tissues and stained with apical markers (i.e., Par6, Ezrin, and aPKC), tight junction markers (ZO1), and basolateral markers (E-Cadherin and Dlg1). For each cell in a mammary duct or lesion, we traced the plasma membrane encircling the nucleus and scored it as polarized or not polarized. To be considered polarized in our study, cells met all of the following criteria: (1) Both apical and basolateral membrane markers were detected in the cell. (2) The apical marker was enriched on a part of the membrane and excluded from another part of the membrane. (3) The basolateral marker was enriched on a part of the membrane and excluded from another part of the membrane. (4) Enrichment of apical proteins and enrichment of basolateral proteins on regions of the plasma membrane were mutually exclusive. A protein was considered enriched if the intensity was higher on the membrane than in the cytoplasm of that cell. A protein was considered excluded from the membrane if the intensity at the membrane was equal to or less than the cytoplasmic intensity in that cell. Nonpolarized cells were considered to have basolateral Par6 if cells expressed overlapping Par6 and E-cadherin uniformly on the plasma membrane. Nonpolarized cells were considered to have high cytoplasmic Par6 if the cytoplasmic intensity was greater than twofold higher than the average cytoplasmic intensity of Par6 in normal duct cells. Measurements were performed manually using ImageJ on tissue sections stained in parallel and imaged sequentially using identical microscope settings. For the human samples, the percentage of cells with apical-basal membrane polarity was determined from nine normal, six $\mathrm{ADH}$, six pure DCIS, 17 DCIS adjacent to IDC, and five IDC sections, all characterized by a pathologist. For the mouse samples, five normal, three hyperplasia/MIN, three early carcinoma, and two late carcinoma sections were examined from two to three different mice each.

To measure the number of polarized and nonpolarized cells that were proliferating, PyMT mouse tumor tissues were stained with the Ki67 proliferation marker and with PKCı to mark the apical membrane. Using ImageJ, counts were performed on four mice with six different fields of view per section at the hyperplasia/MIN stage.

To measure the lumen size and number of cells per lumen, the lumen circumference determined by the apical Par6 signal was measured using the polygon selection tool in ImageJ. We examined 31 images of human sections and $>11$ images from at least three mice at different stages of progression.
To measure the spindle orientation of dividing cells, sections from FVB (control) and PyMT mice 4 wk after palpation were stained for phospho-Histone-H3 to mark dividing cells and with a-tubulin to visualize the spindle pole. The lumen was used as the planar reference, and only dividing cells in regions that were not stratified were measured. Using ImageJ, a line was drawn tangent to the lumen, and another was drawn through the spindle poles; the resulting angle was measured. We measured the angles of 46 dividing cells from seven PyMT mice and 11 dividing cells from six glands from three FVB mice.

The phospho-myosin II and RhoA-GTP intensity was measured from tissue sections from three mice or five patients per condition, with three to 15 images each. For mice, noninduced mammary glands were compared with tissues following $2 \mathrm{wk}$ of doxycycline treatment to induce PyMT expression. Human patient breast lesions were examined for cases containing $\mathrm{ADH}$ and DCIS in the same sample. Phospho-myosin II or RhoA-GTP was costained with Par6 to identify the apical membrane. The average pixel intensity of the phospho-myosin II or RhoA-GTP channel along the apical membrane was measured using the polyline measurement tool in ImageJ.

Circularity was calculated using the shape descriptors feature in Image J measurements and given by the formula circularity = $3 A / 4 \pi r^{2}$, where $A$ is area and $r$ is radius.

\section{Mammary epithelial cell isolation}

Mammary glands from 8- to 12-wk-old mice were chopped and added to digestion medium (10 mL of phenol-free DMEM/F12, $1 \times$ penicillin/streptomycin, $50 \mu \mathrm{g} / \mathrm{mL}$ gentamycin, $2 \mathrm{mg} / \mathrm{mL}$ collagenase A, $600 \mathrm{U} / \mathrm{mL}$ nystatin) for $1-1.5 \mathrm{~h}$. The epithelial cells were then purified into pure epithelial organoids by sequential washes in PBS + 5\% FBS and centrifugations at $1500 \mathrm{rpm}$ for 15 sec. To obtain single cells, the organoids were resuspended in $0.25 \%$ trypsin for $20 \mathrm{~min}$ at $37^{\circ} \mathrm{C}$. The trypsin was then quenched with FBS, and the cells were resuspended in medium. Finally, single epithelial cells were passed through a $40-\mu \mathrm{m}$ mesh to eliminate cell clusters.

\section{Ex vivo 3D organotypic culture}

Following primary mammary epithelial cell extraction, cells were plated in an eight-well chambered coverglass (LabTek II, ThermoFisher Scientific, 155409) or eight-well $\mu$-slide (Ibidi, 80826 ) at a density of 5000 cells per well on top of a layer of $100 \%$ GelTrex (ThermoFisher Scientific, A1413202) in medium supplemented with $2 \%$ GelTrex. The organotypic medium consisted of Epicult-B mouse medium (Stem Cell Technologies, 05610), knockout serum replacement (Gibco, 10828010), penicillin/streptomycin, $10 \mathrm{ng} / \mathrm{mL}$ EGF, $25 \mu \mathrm{g} / \mathrm{mL}$ insulin, and $1 \mu \mathrm{g} / \mathrm{mL}$ hydrocorticone. Cysts formed after 5-7 d in culture. The PyMT oncogene was induced with $2 \mu \mathrm{g} / \mathrm{mL}$ doxycycline for an additional 5-10 d. To categorize the different phenotypes, the assay was performed on three different noninduced MIC mice; the cysts were grown for $8 \mathrm{~d}$ and then fixed with $4 \%$ PFA at days $0,2,4$, 6 , and 8 after doxycycline addition. 3D structures were stained with fluorescent phalloidin to mark the apical membrane, and nuclei were labeled with Hoechst 33342. Images of all of the cysts (or up to 50 images per well) were taken and categorized manually in the different categories according to two criteria: shape of the lumen (single prominent lumen or collapsed microlumen) and layering of the cells around the lumen (monolayer, multilayer, and full/collapsed cyst).

To prepare cells for live imaging, lentivirus containing cDNA for mCherry-H2B was used to infect cells before embedding and 
mark the nuclei. Spindle orientation of dividing cells was then measured using ImageJ.

To knock down p190-B (Arhgap5), shRNA-containing lentivirus was used to infect the single cells before embedding. The shRNAs used were purchased from ThermoFisher Scientific (sh1p190B TRCN0000012703, sh2p190B TRCN0000012704, sh3p190B TRCN0000012705, sh4p190B TRCN0000012706, and sh5p190B TRCN0000012707), and a nontargeting scrambled shRNA was used as a control. Cells were mixed with the lentivirus in medium at a multiplicity of infection (MOI) of 10 and were left spinning at $300 \mathrm{rpm}$ for $3 \mathrm{~h}$ at room temperature. The infected cells were then plated with the virus-containing medium on top of a layer of $100 \%$ GelTrex in medium supplemented with $2 \%$ GelTrex. The medium was changed after $3 \mathrm{~d}$ of plating and then every $2 \mathrm{~d}$ until the cells were fixed. PyMT was induced using 2 $\mu \mathrm{g} / \mathrm{mL}$ doxycycline $6-8 \mathrm{~d}$ after plating for an additional 5-7 $\mathrm{d}$.

\section{Live imaging}

Organotypic cultures were treated with $2 \mu \mathrm{g} / \mathrm{mL}$ doxycycline or water, and imaging was started immediately using an automated Zeiss LSM700 confocal microscope with a $20 \times 0.8$ NA objective lens. $Z$-stacked image series were collected every $15 \mathrm{~min}$ for 72-96 h. Noninduced samples were used as negative controls and collected in parallel with induced samples using an automated stage.

\section{Orthotopic transplants}

Following primary mammary epithelial cell extraction and infection, cells were added to mammosphere medium plated in suspension for $3 \mathrm{~d}$. The mammosphere medium consisted of Epicult-B mouse medium (Stem Cell Technologies, 05610), knockout serum replacement (Gibco, 10828010), penicillin/ streptomycin, $20 \mathrm{ng} / \mathrm{mL}$ EGF, $20 \mathrm{ng} / \mathrm{mL} \mathrm{FGF}$, and $4 \mu \mathrm{g} / \mathrm{mL}$ heperan sulfate. Next, the cells were spun down, resuspended in PBS with $10 \%$ GelTrex and 10\% Trypan blue, and injected into the cleared mammary fat pad of a 3-wk-old FVB female mouse. Each injection was $10-12 \mu \mathrm{L}$ in volume.

\section{Statistical analysis}

Comparisons of multiple means was performed by ANOVA using Tukey's post-hoc test in general or Sidak's post-hoc test specifically for the shp190-B knockdown cysts. An a of 0.05 was used for determining statistical significance. Comparison of two unpaired independent means was performed using a Student's $t$ test. Statistics were determined using Excel, SPSS, and GraphPad Prism. All images are representative of more than five fields from at least three mice or five human tumor samples.

\section{Acknowledgments}

We thank Jo-Ann Bader, the McGill Histology Core, and Siying Li for technical assistance. R.H. was supported by the Fonds Recherche du Quebec-Santé award. S.J.C. was supported by Defi Canderel and Karassik Family Foundation awards. L.M. is a Fonds Recherche du Quebec-Santé Research Scholar (Junior 2). This work was supported by a Komen Career Catalyst grant (CCR15331358) and grants from the Cancer Research Society (63724) and the Quebec Breast Cancer Foundation (64919) to L. $\mathrm{M}$. Confocal imaging was made possible with an equipment grant funded by the Canada Foundation for Innovation to L.M.

\section{References}

Allred DC, Wu Y, Mao S, Nagtegaal ID, Lee S, Perou CM, Mohsin SK, O'Connell P, Tsimelzon A, Medina D. 2008. Ductal carcinoma in situ and the emergence of diversity during breast cancer evolution. Clin Cancer Res 14: 370-378.

Aranda V, Haire T, Nolan ME, Calarco JP, Rosenberg AZ, Fawcett JP, Pawson T, Muthuswamy SK. 2006. Par6-aPKC uncouples ErbB2 induced disruption of polarized epithelial organization from proliferation control. Nat Cell Biol 8: 1235-1245.

Archibald A, Mihai C, Macara IG, McCaffrey L. 2015. Oncogenic suppression of apoptosis uncovers a Racl/JNK proliferation pathway activated by loss of Par3. Oncogene 34: 3199-3206.

Bombonati A, Sgroi DC. 2011. The molecular pathology of breast cancer progression. J Pathol 223: 308-318.

Chakravarty G, Roy D, Gonzales M, Gay J, Contreras A, Rosen JM. 2000. P190-B, a Rho-GTPase-activating protein, is differentially expressed in terminal end buds and breast cancer. Cell Growth Differ 11: 343-354.

Cheung KJ, Gabrielson E, Werb Z, Ewald AJ. 2013. Collective invasion in breast cancer requires a conserved basal epithelial program. Cell 155: 1639-1651.

Danes CG, Wyszomierski SL, Lu J, Neal CL, Yang W, Yu D. 2008. 14-3-3 $\zeta$ down-regulates p53 in mammary epithelial cells and confers luminal filling. Cancer Res 68: 1760-1767.

Ellis IO. 2010. Intraductal proliferative lesions of the breast: morphology, associated risk and molecular biology. Mod Pathol 23: S1-S7.

Feigin ME, Akshinthala SD, Araki K, Rosenberg AZ, Muthuswamy LB, Martin B, Lehmann BD, Berman HK, Pietenpol JA, Cardiff RD, et al. 2014. Mislocalization of the cell polarity protein scribble promotes mammary tumorigenesis and is associated with basal breast cancer. Cancer Res 74: 3180-3194.

Fluck MM, Schaffhausen BS. 2009. Lessons in signaling and tumorigenesis from polyomavirus middle $\mathrm{T}$ antigen. Microbiol Mol Biol Rev 73: 542-563.

Fogg VC, Liu CJ, Margolis B. 2005. Multiple regions of Crumbs3 are required for tight junction formation in MCF10A cells. I Cell Sci 118: 2859-2869.

Global Burden of Disease Cancer Collaboration 2015. The global burden of cancer 2013. JAMA Oncol 1: 505-527.

Godde NJ, Sheridan JM, Smith LK, Pearson HB, Britt KL, Galea RC, Yates LL, Visvader JE, Humbert PO. 2014. Scribble modulates the MAPK/Fral pathway to disrupt luminal and ductal integrity and suppress tumour formation in the mammary gland. PLoS Genet 10: e1004323.

Guy CT, Cardiff RD, Muller WJ. 1992. Induction of mammary tumors by expression of polyomavirus middle $\mathrm{T}$ oncogene: a transgenic mouse model for metastatic disease. Mol Cell Biol 12: 954-961.

Halaoui R, McCaffrey L. 2015. Rewiring cell polarity signaling in cancer. Oncogene 34: 939-950.

Hannemann J, Velds A, Halfwerk JB, Kreike B, Peterse JL, van de Vijver MJ. 2006. Classification of ductal carcinoma in situ by gene expression profiling. Breast Cancer Res 8: R61.

Haque R, Ahmed SA, Inzhakova G, Shi J, Avila C, Polikoff J, Bernstein L, Enger SM, Press MF. 2012. Impact of breast cancer subtypes and treatment on survival: an analysis spanning two decades. Cancer Epidemiol Biomarkers Prev 21: 18481855.

Heckman-Stoddard BM, Vargo-Gogola T, McHenry PR, Jiang V, Herrick MP, Hilsenbeck SG, Settleman J, Rosen JM. 2009. Haploinsufficiency for p190B RhoGAP inhibits MMTV-Neu tumor progression. Breast Cancer Res 11: R61. 
Herschkowitz JI, Simin K, Weigman VI, Mikaelian I, Usary J, Hu Z, Rasmussen KE, Jones LP, Assefnia S, Chandrasekharan S, et al. 2007. Identification of conserved gene expression features between murine mammary carcinoma models and human breast tumors. Genome Biol 8: R76.

Huang L, Muthuswamy SK. 2010. Polarity protein alterations in carcinoma: a focus on emerging roles for polarity regulators. Curr Opin Genet Dev 20: 41-50.

Huebner RJ, Ewald AJ. 2014. Cellular foundations of mammary tubulogenesis. Semin Cell Dev Biol 31: 124-131.

Huebner RJ, Lechler T, Ewald AJ. 2014. Developmental stratification of the mammary epithelium occurs through symmetrybreaking vertical divisions of apically positioned luminal cells. Development 141: 1085-1094.

Jechlinger M, Podsypanina K, Varmus H. 2009. Regulation of transgenes in three-dimensional cultures of primary mouse mammary cells demonstrates oncogene dependence and identifies cells that survive deinduction. Genes Dev 23: 1677-1688.

Kos Z, Dabbs DJ. 2016. Biomarker assessment and molecular testing for prognostication in breast cancer. Histopathology 68: 70-85.

Kuipers D, Mehonic A, Kajita M, Peter L, Fujita Y, Duke T, Charras G, Gale JE. 2014. Epithelial repair is a two-stage process driven first by dying cells and then by their neighbours. I Cell Sci 127: 1229-1241.

Lee JL, Streuli CH. 2014. Integrins and epithelial cell polarity. I Cell Sci 127: 3217-3225.

Leung CT, Brugge JS. 2012. Outgrowth of single oncogene-expressing cells from suppressive epithelial environments. $\mathrm{Na}$ ture 482: 410-413.

Lin EY, Jones JG, Li P, Zhu L, Whitney KD, Muller WJ, Pollard JW. 2003. Progression to malignancy in the polyoma middle $T$ oncoprotein mouse breast cancer model provides a reliable model for human diseases. Am J Pathol 163: 2113-2126.

Liu P, Cheng H, Santiago S, Raeder M, Zhang F, Isabella A, Yang J, Semaan DJ, Chen C, Fox EA, et al. 2011. Oncogenic PIK3CAdriven mammary tumors frequently recur via PI3K pathwaydependent and PI3K pathway-independent mechanisms. Nat Med 17: 1116-1120.

Lopez-Garcia MA, Geyer FC, Lacroix-Triki M, Marchio C, ReisFilho JS. 2010. Breast cancer precursors revisited: molecular features and progression pathways. Histopathology 57: 171-192.

Maglione JE, Moghanaki D, Young LJ, Manner CK, Ellies LG, Joseph SO, Nicholson B, Cardiff RD, MacLeod CL. 2001. Transgenic Polyoma middle-T mice model premalignant mammary disease. Cancer Res 61: 8298-8305.

Mailleux AA, Overholtzer M, Brugge JS. 2008. Lumen formation during mammary epithelial morphogenesis: insights from in vitro and in vivo models. Cell Cycle 7: 57-62.

Mao Y, Tournier AL, Hoppe A, Kester L, Thompson BJ, Tapon N. 2013. Differential proliferation rates generate patterns of mechanical tension that orient tissue growth. EMBO $J$ 32: 2790-2803.

Martin AC, Goldstein B. 2014. Apical constriction: themes and variations on a cellular mechanism driving morphogenesis. Development 141: 1987-1998.

McCaffrey LM, Macara IG. 2009. The Par3/aPKC interaction is essential for end bud remodeling and progenitor differentiation during mammary gland morphogenesis. Genes Dev 23: 1450-1460.

McCaffrey LM, Montalbano J, Mihai C, Macara IG. 2012. Loss of the Par3 polarity protein promotes breast tumorigenesis and metastasis. Cancer Cell 22: 601-614.
Nakajima H, Tanoue T. 2011. Lulu2 regulates the circumferential actomyosin tensile system in epithelial cells through p114RhoGEF. J Cell Biol 195: 245-261.

Nguyen-Ngoc KV, Cheung KJ, Brenot A, Shamir ER, Gray RS, Hines WC, Yaswen P, Werb Z, Ewald AJ. 2012. ECM microenvironment regulates collective migration and local dissemination in normal and malignant mammary epithelium. Proc Natl Acad Sci 109: E2595-E2604.

Pradeep CR, Kostler WJ, Lauriola M, Granit RZ, Zhang F, JacobHirsch J, Rechavi G, Nair HB, Hennessy BT, Gonzalez-Angulo AM, et al. 2012. Modeling ductal carcinoma in situ: a HER2Notch3 collaboration enables luminal filling. Oncogene 31: 907-917.

Rao T, Ranger JJ, Smith HW, Lam SH, Chodosh L, Muller WJ. 2014. Inducible and coupled expression of the polyomavirus middle $\mathrm{T}$ antigen and Cre recombinase in transgenic mice: an in vivo model for synthetic viability in mammary tumour progression. Breast Cancer Res 16: R11.

Rodriguez-Boulan E, Macara IG. 2014. Organization and execution of the epithelial polarity programme. Nat Rev Mol Cell Biol 15: 225-242.

Sgroi DC. 2010. Preinvasive breast cancer. Annu Rev Pathol 5: 193-221.

Shore AN, Chang CH, Kwon OJ, Weston MC, Zhang M, Xin L, Rosen JM. 2016. PTEN is required to maintain luminal epithelial homeostasis and integrity in the adult mammary gland. Dev Biol 409: 202-217.

Sinn HP, Elsawaf Z, Helmchen B, Aulmann S. 2010. Early breast cancer precursor lesions: lessons learned from molecular and clinical studies. Breast Care (Basel) 5: 218-226.

Tang Y, Wang Y, Kiani MF, Wang B. 2016. Classification, treatment strategy, and associated drug resistance in breast cancer. Clin Breast Cancer 16: 335-343.

Taraseviciute A, Vincent BT, Schedin P, Jones PL. 2010. Quantitative analysis of three-dimensional human mammary epithelial tissue architecture reveals a role for tenascin-C in regulating c-met function. Am J Pathol 176: 827-838.

Terry SJ, Zihni C, Elbediwy A, Vitiello E, Leefa Chong San IV, Balda MS, Matter K. 2011. Spatially restricted activation of RhoA signalling at epithelial junctions by p114RhoGEF drives junction formation and morphogenesis. Nat Cell Biol 13: 159-166.

Vargo-Gogola T, Heckman BM, Gunther EJ, Chodosh LA, Rosen JM. 2006. P190-B Rho GTPase-activating protein overexpression disrupts ductal morphogenesis and induces hyperplastic lesions in the developing mammary gland. Mol Endocrinol 20: 1391-1405.

Venugopalan G, Camarillo DB, Webster KD, Reber CD, Sethian JA, Weaver VM, Fletcher DA, El-Samad H, Rycroft $\mathrm{CH}$. 2014. Multicellular architecture of malignant breast epithelia influences mechanics. PLoS One 9: e101955.

Xue B, Krishnamurthy K, Allred DC, Muthuswamy SK. 2013. Loss of Par3 promotes breast cancer metastasis by compromising cell-cell cohesion. Nat Cell Biol 15: 189-200.

Zandvakili I, Lin Y, Morris JC, Zheng Y. 2017. Rho GTPases: antior pro-neoplastic targets? Oncogene 36: 3213-3222.

Zhan L, Rosenberg A, Bergami KC, Yu M, Xuan Z, Jaffe AB, Allred C, Muthuswamy SK. 2008. Deregulation of scribble promotes mammary tumorigenesis and reveals a role for cell polarity in carcinoma. Cell 135: 865-878.

Zhang M, Lee AV, Rosen JM. 2017. The cellular origin and evolution of breast cancer. Cold Spring Harb Perspect Med 7: a027128. 


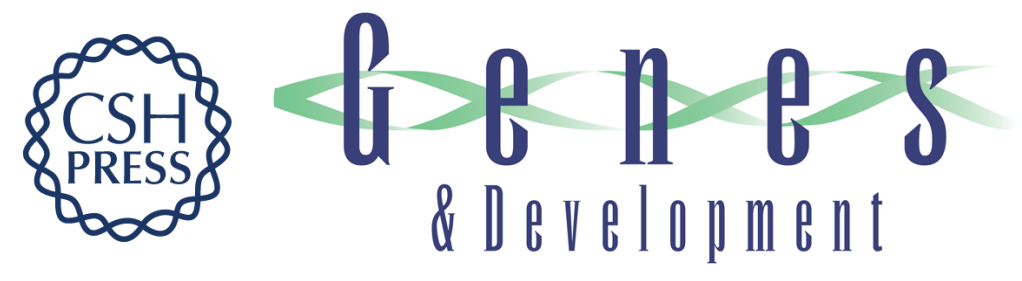

\section{Progressive polarity loss and luminal collapse disrupt tissue organization in carcinoma}

Ruba Halaoui, Carlis Rejon, Sudipa June Chatterjee, et al.

Genes Dev. 2017, 31: originally published online September 8, 2017

Access the most recent version at doi:10.1101/gad.300566.117

\section{Supplemental http://genesdev.cshlp.org/content/suppl/2017/09/08/gad.300566.117.DC1 Material}

References This article cites 83 articles, 32 of which can be accessed free at: http://genesdev.cshlp.org/content/31/15/1573.full.html\#ref-list-1

Creative This article is distributed exclusively by Cold Spring Harbor Laboratory Press for the first Commons six months after the full-issue publication date (see

License http://genesdev.cshlp.org/site/misc/terms.xhtml). After six months, it is available under a Creative Commons License (Attribution-NonCommercial 4.0 International), as described at http://creativecommons.org/licenses/by-nc/4.0/.

Email Alerting Receive free email alerts when new articles cite this article - sign up in the box at the top Service right corner of the article or click here.

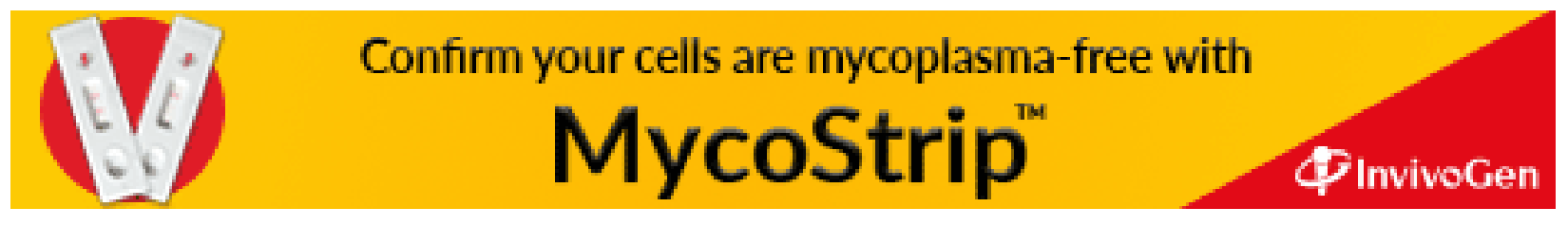

